\title{
New dimensions in palliative care: a palliative approach to neurodegenerative diseases and final illness in older people
}

\author{
Linda J Kristjanson, Christine Toye and Sky Dawson
}

UNTIL RECENTLY, PALliative CARE has focused on the needs of patients with cancer and their families. However, of late, palliative care has become engaged in the care of people with other progressive, terminal conditions, such as neurodegenerative diseases. For these people, a palliative care approach, rather than specialist palliative care services and palliative interventions, may offer the most benefit. This approach is also most relevant to the final illness of elderly people dying from conditions other than cancer.

\section{Palliative care: variations on a theme}

To understand the potential contribution of palliative care in conditions other than cancer, we must distinguish the different types of palliative care.

Specialist palliative care services are important in the care of a minority of people whose deaths are expected. These services may accept prime responsibility for care, or work indirectly through advising patients' professional carers. They provide physical, psychological, social and spiritual support and comprise a broad range of disciplines, including medicine, nursing, social work, pastoral care, physiotherapy, occupational therapy, pharmacy and related specialties. $^{1}$

Palliative interventions are non-curative interventions, implemented by workers in disciplines other than palliative care, which aim to control symptoms and improve quality of life. These interventions include palliative radiotherapy, surgical procedures and anaesthetic techniques for pain relief, ${ }^{1}$ and may be used at any time during an illness.

The palliative care approach aims to improve the quality of life of individuals facing life-threatening illness and of their families, by preventing and relieving suffering through early identification, assessment and treatment of pain and other problems - physical, psychosocial and spiritual. ${ }^{2}$ This definition implies that people with progressive fatal diseases other than cancer may benefit from a palliative approach, with a positive and open attitude towards death and dying. This approach facilitates identification of the wishes of patients and their families about care throughout a period of declining health and especially during end-of-life care.

School of Nursing and Public Health, Edith Cowan University, Perth, WA.

Linda J Kristjanson, PhD, Professor; Christine Toye, PhD, Postdoctoral Fellow;

Sky Dawson, MSc, Research Officer.

Reprints: Professor Linda J Kristjanson, School of Nursing and Public Health, Edith Cowan University, Pearson Street, Churchlands, WA 6018. I.kristjanson@ecu.edu.au

\section{ABSTRACT}

- A palliative care approach has much to offer people in the advanced stages of neurodegenerative diseases, as well as elderly people dying from diseases other than cancer.

- Palliative care can be part of the treatment repertoire of any health worker, supported by intermittent consultation or referral to specialist palliative care services (eg, for management of neuropathic pain).

- A palliative care approach encourages a focus on pain and symptom management, and prompts more open communication about end-of-life issues.

- This approach recruits as necessary the expertise of specialists and multidisciplinary teams to encourage a flexible, responsive service.

- Home carers and healthcare providers require education to ensure a palliative approach that meets the physical, psychological, spiritual and social challenges facing patients and their families, and enhances dignity and quality of life.

MJA 2003; 179: \$41-\$43

A palliative approach can be a particularly helpful component of care for patients with neurodegenerative diseases, as we will describe. It is also the appropriate approach to care of the very elderly when they are approaching death.

\section{Palliative care in neurodegenerative diseases}

People living with neurological diseases such as motor neurone disease, multiple sclerosis, Huntington's disease and Parkinson's disease face long-term physical and psychosocial challenges. These diseases are incurable and progressive, with duration in years or decades, including long periods of dependency. A range of technologies and healthcare options may ensure adequate nutrition, communication, cardiac and respiratory functioning, bowel motility and skin integrity, but all require learning and adjustment by the individual and family. The psychological and social factors of managing living with a neurodegenerative disease are as important as physical care. Healthy recovery of family members after their eventual bereavement is also essential.

Neurodegenerative diseases may evoke negative attitudes in many health professionals, which may be readily transmitted to patients and their families. ${ }^{3}$ Our inability to significantly alter disease progression is sometimes interpreted to mean that nothing more can be done, ${ }^{4}$ but is precisely the reason that we must do everything possible to alleviate 
symptoms and offer appropriate psychological and spiritual support. This is where a palliative approach has much to offer.

Although many neurodegenerative diseases are relatively rare, many of their associated symptoms are common among other patients (eg, pain, breathlessness, constipation and insomnia), and their routine management should fall within the scope of all competent practitioners. ${ }^{3}$ More complex issues, such as nutritional support using enteral feeding, management of respiratory function with a view to ventilator support, or management of neuropathic pain, may require consultation or referral to specialist palliative care services. $^{3}$

\section{Pain management and symptom relief}

Pain is common in neurodegenerative diseases such as multiple sclerosis and motor neurone disease. In multiple sclerosis, about half of all patients have pain, ${ }^{5,6}$ with prevalence increasing with longer disease duration and greater severity of neurological symptoms. ${ }^{7,8}$ The most common causes are dysaesthesia and muscle spasms. ${ }^{7,8}$ Because of the high frequency of neuropathic pain, adjuvant drugs have a major role, and consultation with palliative care specialists may be helpful. ${ }^{3}$ Unfortunately, there is evidence that these patients are often undertreated. ${ }^{7,9}$

Similarly, in motor neurone disease, recent studies indicate that about three-quarters of patients have pain. ${ }^{10}$ Evidence suggests it is underdiagnosed and undertreated, and that a palliative approach may direct practitioners to more focused and effective assessment of pain. ${ }^{10,11}$ Related symptoms may also be undertreated. For example, a study comparing 124 patients with motor neurone disease and a group of cancer patients, all admitted to the same hospice, found that although pain was less prevalent in the patients with motor neurone disease (58\% v 69\%), insomnia and constipation were more prevalent $(48 \% \mathrm{v} 29 \%$ and $65 \% \mathrm{v}$ $48 \%$, respectively), while breathlessness was equally prevalent $(47 \% \mathrm{v} 50 \%) .{ }^{10}$ At referral, only 15 patients with motor neurone disease were receiving an opioid, although 71 had uncontrolled pain. During their admission, 109 patients with motor neurone disease received an opioid on at least one occasion. The mean dose was morphine $30 \mathrm{mg}$ orally every 24 hours, and the mean duration of treatment was 58 days (SD, 18.5). This study clearly indicates that morphine may be effective in relieving much of these patients' distress. ${ }^{3}$

A palliative approach involves attention to the full range of symptoms that patients may encounter. It discourages complacency about their inevitability, and encourages active efforts to assess and intervene wherever possible.

\section{Psychological support}

Although palliative care providers are best known for their expertise in symptom management, the palliative care approach has a primary focus on hope, quality of life and dignity. It prompts more open communication about endof-life issues, ${ }^{12}$ emphasising good communication between patients and their carers, comfort with discussing loss and impending death, and attention to signs of depression and anxiety. This is especially relevant in neurodegenerative diseases such as Parkinson's disease and Huntington's disease.

In Parkinson's disease, psychological and communication problems include depression, personality changes, sleep disturbances and dementia. ${ }^{3}$ Huntington's disease also affects cognition and personality as well as movement. ${ }^{3}$ Cognitive impairment is usually apparent at the onset of the movement disorder and follows a progressive and unremitting course. ${ }^{3}$ Patients with this disease must cope with significant feelings of loss and fear of removal of their dignity. As it is a genetic disease, family members have to cope not only with the cognitive and behavioural changes in their relative, but also with the distress of "rehearsing" their own future illness if they are genetically at risk. Long-term care needs in this disease are considerable, and decisions to move the patient to a residential care facility may be fraught with guilt, loss and grief. ${ }^{13}$

Neurodegenerative diseases are also commonly accompanied by mood disturbances. In multiple sclerosis, correlations between disease activity and depression have been reported. ${ }^{3,14}$ Similarly, in motor neurone disease, correlations between increased physical dependency and depression, and between speech impairment and anxiety, have been found. ${ }^{15}$ Psychological support and preparation for future progression of these conditions is an aspect of a palliative care approach that may be particularly relevant.

In addition, neurodegenerative diseases may affect articulation, and speech may become unintelligible. The inability to communicate can cause intense frustration for all parties. ${ }^{3}$ A commitment to identifying ways of communicating (eg, communication boards and computerised methods) and anticipation of communication difficulties are essential. Although the techniques of enhancing communication are not unique to a palliative approach, the attention given to communication is heightened when palliation is the primary goal of care. As well, a palliative approach will encourage different communication content, incorporating discussion about loss and grief, which may help alleviate psychological distress. Loss of communication due to physical or cognitive changes can be considered a type of "social death", and families and patients need help to come to terms with this loss and the isolation it causes.

The dementia associated with Huntington's and Parkinson's diseases may compromise not only communication, but also assessment of pain and management of distress. There is evidence that patients with cognitive impairments are less likely to receive analgesia. ${ }^{16}$ Agitated behaviours may be difficult to interpret, and medications may consequently be over- or underused. Involvement of palliative care specialists at this stage of illness may help discern the nature of distress.

Dementia is also commonly associated with advanced age. Alzheimer's disease, the most common neurodegenerative disease, responsible for $50 \%-70 \%$ of cases of dementia, ${ }^{17}$ is strongly correlated with ageing. ${ }^{18}$ Because of comorbidities, 
illness in older people has the potential to cause exceptionally high levels of symptom distress, which are particularly difficult to alleviate in the presence of dementia. In addition, the often severe and long-term nature of functional incapacity in the elderly, and the fact that family carers also tend to be elderly, may necessitate the use of institutional care. A palliative care approach can guide patients, families and formal carers in their management of the downward trajectories of final illnesses during ageing.

\section{Barriers to a palliative approach}

Although many clinicians may use some elements of a palliative approach in the care of individuals with neurodegenerative diseases and the elderly at the end of life, the explicit and rigorous use of a palliative approach has not been routine. Reasons may include the belief that a palliative approach should only be offered in the final stages of an illness, difficulty recognising when patients are terminally ill, therapeutic "ennui" (disengagement or difficulty in imagining alternative approaches when active treatment is not available), discomfort in discussing end-of-life issues, the belief that symptoms are acceptable or inevitable, ${ }^{19}$ and ignorance about palliative care.

Inclusion of a palliative approach should not be delayed until the end stages of an illness. In fact, incorporating a palliative care approach in the early stages may alleviate physical and psychological distress at that time. It can provide a focus on active comfort care and a positive approach to ameliorating symptoms and distress when therapeutic "ennui" emerges. A commitment to ensuring comfort and relief of suffering can assist patients and families to know that they are actively supported as the illness progresses.

\section{Conclusions}

The need for more widespread dissemination of palliative care knowledge to general practitioners, nurses, and allied health professionals is being recognised. For example, the nationally funded Australian Palliative Aged Care project is currently developing a training program in palliative care for the many tiers of staff working in aged care. ${ }^{20}$ This type of focused competency development in palliative care might be offered to other health professionals, such as those who work with patients with neurodegenerative diseases.

\section{Competing interests}

None identified.

\section{References}

1. Addington-Hall J, Higginson IJ. Palliative care for non-cancer patients. Oxford: Oxford University Press, 2001

2. World Health Organization. Palliative care. Available at: www.who.int/hiv/topics/ palliative/PalliativeCare/en/ (accessed Jul 2003).

3. O'Brien T. Neurodegenerative disease. In: Addington-Hall J, Higginson IJ, editors. Palliative care for non-cancer patients. Oxford: Oxford University Press, 2001: 44-53.
4. Norris EH. Motor neurone disease - treating the untreated. BMJ 1992; 304: 459-460.

5. Houtchens MK, Richert JR, Sami A, Rose JW. Open label gabapentin treatment for pain in multiple sclerosis. Mult Scler 1997; 3: 250-253.

6. Moulin DE. Pain in multiple sclerosis. Neurol Clin 1989; 7: 321-331.

7. Archibald CJ, McGrath PG, Ritvo PG, et al. Pain prevalence, severity and impact in a clinical sample of multiple sclerosis patients. Pain 1994; 58: 89-93.

8. Moulin DE, Foley KM, Ebers GC. Pain syndromes in multiple sclerosis. Neurology 1988; 38: 1830-1834.

9. Knudsen SE, Jensen K. Acute and chronic pain syndromes in multiple sclerosis. Acta Neurol Scand 1991; 84: 197-200

10. O'Brien T, Kelly M, Saunders C. Motor neurone disease: a hospice perspective. BMJ 1992; 304: 471-473.

11. Oliver D. The quality of care and symptom control - the effects of the terminal phase of ALS/MND. J Neurol Sci 1996; 139 Suppl: 134-136.

12. Addington-Hall J, Higginson I. Discussion. In: Addington-Hall J, Higginson IJ, editors. Palliative care for non-cancer patients. Oxford: Oxford University Press, 2001: 278-286.

13. Abudi S, Bar-Tal Y, Ziv L, Fish M. Parkinson's disease symptoms: patients' perceptions. J Adv Nurs 1997; 25: 54-59

14. Tedman BM, Young CA, Williams IR. Assessment of depression in patients with motor neurone disease and other neurologically disabling illness. J Neurol Sci 1997; 152 Suppl 1: S75-S79.

15. Hogg KE, Goldstein LJ, Leigh PN. The psychological impact of motor neurone disease. Psychol Med 1994, 24: 625-632.

16. Scherder EA, Bouma A. Is decreased use of analgesics in Alzheimer's disease due to a change in the affective component of pain. Alzheimer Dis Assoc Disord 1997; 11: 171-174.

17. Access Economics. The dementia epidemic: economic impact and positive solutions for Australia. Report prepared for Alzheimer's Australia. Canberra: Alzheimer's Australia, 2003. Available at www.alzheimers.org.au (accessed Jun 2003).

18. Australian Institute of Health and Welfare and Australian Department of Health and Ageing. Older Australia at a glance. 3rd edition. Canberra: AlHW/DHA, 2002. (AlHW Catalogue No. AGE 25.)

19. Dunlop R. Specialist palliative care and non-malignant diseases. In: AddingtonHall J, Higginson IJ, editors. Palliative care for non-cancer patients. Oxford: Oxford University Press, 2001: 189-197.

20. Australian Palliative Aged Care Project. The APAC Project, 2003. Available at: www.apacproject.org (accessed Jul 2003).

(Received 28 May 2003, accepted 31 Jul 2003) $\square$

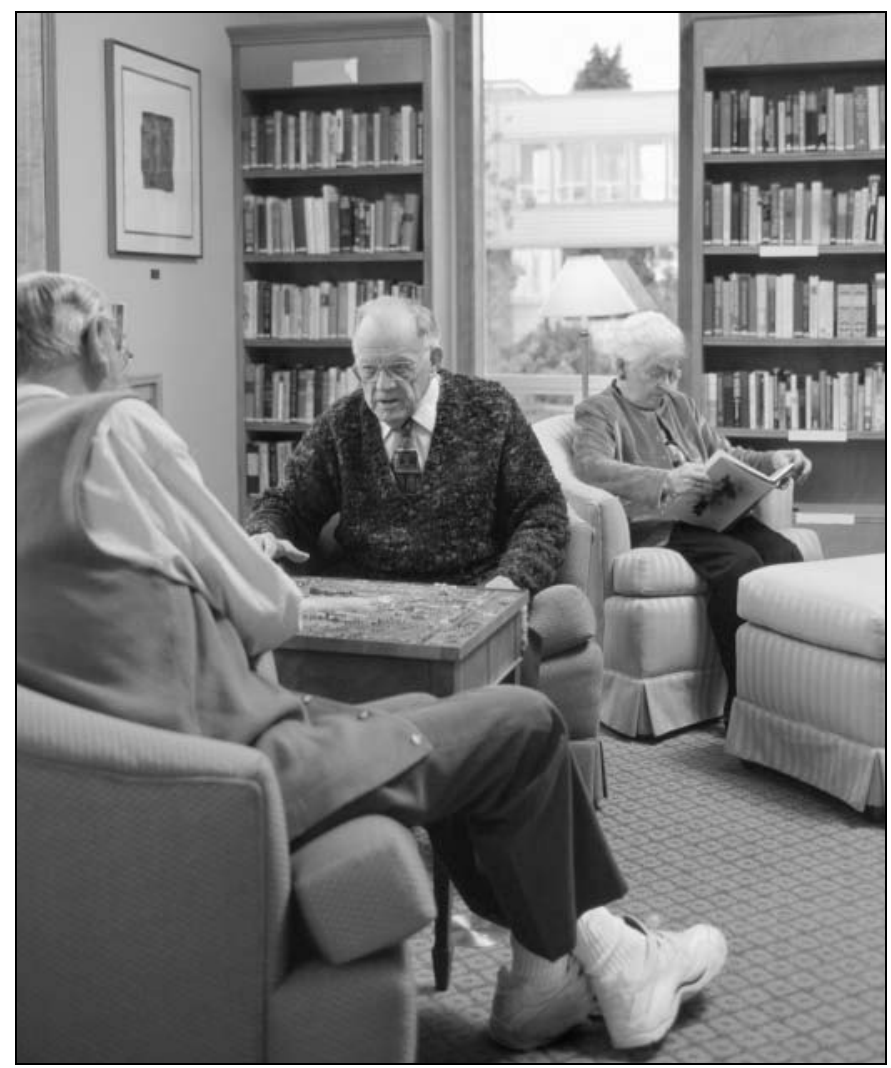

